\title{
Ytterbium triflate (and trimethylsilyl triflate) catalyzed isomerization of glycidic esters to $\alpha$-hydroxy- $\beta, \gamma$-unsaturated esters and their conversion into cyclopentanoids using Johnson-Claisen rearrangement ${ }^{1}$
}

\author{
R. Kumareswaran, ${ }^{\text {a }}$ Shatrughan P. Shahi, ${ }^{\text {a }}$ Shikha Rani, ${ }^{a}$ Anshu Gupta, ${ }^{\text {a }}$ K. P. \\ Madhusudanan ${ }^{\mathrm{b}}$, and Yashwant D. Vankar ${ }^{\mathrm{a} *}$ \\ ${ }^{a}$ Department of Chemistry, Indian Institute of Technology, Kanpur 208 016, India, and \\ ${ }^{b}$ Regional Sophisticated Instrumentation Centre, Central Drug Research Institute, \\ Lucknow 226 001, India \\ E-mail:vankar@iitk.ac.in
}

This paper is respectfully dedicated to Prof. S.V. Kessar on the occasion of his $70^{\text {th }}$ birthday (received 19 Apr 02; accepted 07 Aug 02; published on the web 15 Aug 02)

\begin{abstract}
A variety of glycidic esters undergo smooth isomerization to the corresponding $\alpha$-hydroxy- $\beta, \gamma-$ unsaturated esters upon reaction with $\mathrm{Yb}(\mathrm{OTf})_{3}$ or TMSOTf. These $\alpha$-hydroxy- $\beta, \gamma$-unsaturated esters undergo Johnson-Claisen rearrangement to appropriately substituted diesters, some of which are converted into cyclopentanoids.
\end{abstract}

Keywords: Glycidic esters, $\quad \mathrm{Yb}(\mathrm{OTf})_{3}, \quad \mathrm{TMSOTf}, \quad \alpha$-hydroxy- $\beta, \gamma$-unsaturated esters, cyclopentanoids, Johnson-Claisen rearrangment

\section{Introduction}

$\alpha$-Hydroxy- $\beta$, $\gamma$-unsaturated esters are readily derived from the corresponding glycidic esters ${ }^{2,3}$ using protic or Lewis acids via their regioselective isomerizations. Out of the two possible isomerizations (path ' $a$ ' and path 'b', Scheme 1) of glycidic esters, the one leading predominantly to $\alpha$-hydroxy- $\beta, \gamma$-unsaturated esters involves use of catalysts such as $\mathrm{H}_{2} \mathrm{SO}_{4}{ }^{3}$ $\mathrm{HCl}^{4}{ }^{4} \mathrm{BF}_{3} \cdot \mathrm{Et}_{2} \mathrm{O},{ }^{5} \mathrm{LiClO}_{4},{ }^{6}$ zeolite $\mathrm{H} \mathrm{ZSM}-5,{ }^{7}$ and Nafion-H. ${ }^{8}$ However, with stronger protic $\left(\mathrm{H}_{2} \mathrm{SO}_{4}\right)^{3}$ or Lewis acids $\left(\mathrm{BF}_{3}\right.$. Et $\left.\mathrm{E}_{2} \mathrm{O}\right){ }^{5}$ varying degrees of both kinds of isomerization are reported and appear to be a function of temperature.

Ytterbium triflate is known ${ }^{9}$ to be a useful mild Lewis acid and has gained importance in recent years. Therefore, it occurred to us that it may permit isomerization of glycidic esters by either path ' $a$ ' or ' $b$.' Indeed, it was found that $\mathrm{Yb}(\mathrm{OTf})_{3}$ readily converts a variety of glycidic 
esters 1 (Scheme 1) into the corresponding $\alpha$-hydroxy- $\beta, \gamma$-unsaturated esters 2 (path ' $b$ ') at room temperature with no trace of $\alpha$-keto esters 3 being formed via path ' $a$ '. Our results are summarized in Table 1. We have also found that trimethylsilyl triflate (TMSOTf) allows similar isomerization (Table 1), albeit, at low temperature $\left(-40^{\circ} \mathrm{C}\right)$ and in some cases giving small amount $(\approx 5-10 \%)$ of $\alpha$-keto esters.

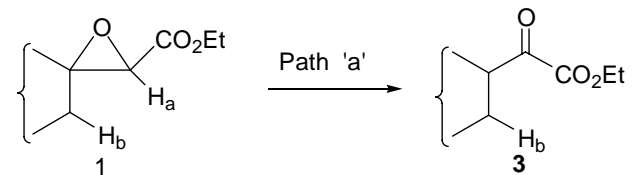

$\downarrow$ Path 'b'

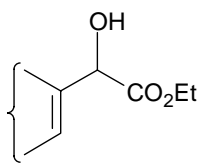

2

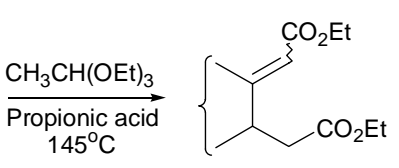

4

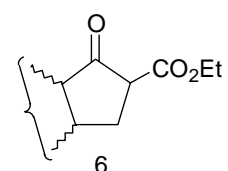

$\mathrm{NaH} / \mathrm{DMSO}$

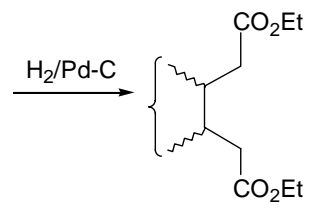

Scheme 1

\section{Results and Discussion}

$\alpha, \beta$-Unsaturated pyruvate esters, obtained via oxidation of $\alpha$-hydroxy- $\beta, \gamma$-unsaturated esters, have been found to be excellent Michael acceptors ${ }^{5}$ and dienophiles in Diels-Alder reactions. ${ }^{5,10}$ We have shown ${ }^{11}$ that $\alpha$-hydroxy- $\beta, \gamma$-unsaturated esters are useful in the preparation of several vinylic epoxides. It was further shown by us ${ }^{12}$ that enzymatic resolution of $\alpha$-hydroxy- $\beta, \gamma-$ unsaturated esters as well as isomerization of (-)-menthyl group bearing glycidic esters eventually lead to the formation of chiral vinylic epoxides. In continuation to explore the potential of $\alpha$-hydroxy- $\beta, \gamma$-unsaturated esters we have now found that they serve as excellent precursors for the synthesis of cyclopentanoids (Scheme 1) via Johnson-Claisen rearrangement, ${ }^{13}$ which occurs in a facile manner to form the corresponding diesters 4 (Table 2). These partly unsaturated diesters upon hydrogenation led to the corresponding fully saturated diesters 5, some of which were converted into cyclopentanoids 6 via Dieckman cyclization. ${ }^{14}$

The saturated diesters 5 were obtained as a mixture of cis-trans isomers with predominance of the trans isomer. These isomers, however, could not be separated by column chromatography. The diester 7c (Scheme 2), derived from cyclopentanone glycidic ester, was reluctant to undergo Dieckman cyclization to form 19 even at higher temperatures due to the trans stereochemistry ${ }^{18}$ at the junction. On the other hand, the corresponding diester 8c from cyclohexanone readily cyclized to form the hydrindane system 20 as a mixture of cis and trans isomers (40:60). ${ }^{18,19}$ The bicyclic system $\mathbf{1 8 \mathbf { a } ^ { 7 }}$ led to the formation of the corresponding diester $\mathbf{1 8 b}$ upon Johnson-Claisen rearrangement via the ketene acetal intermediate $\mathbf{A}$, which subsequently underwent smooth 
hydrogenation followed by Dieckman cyclization to form the angularly fused tricyclic system 21/22 as a $1: 1$ mixture.

In an attempt to make use of this type of cyclopentanoid formation in the synthesis of tricyclopentanoids, the bicyclo (3.3.0) system $23^{20}$ was converted into the corresponding diester 27 via the same sequence of reactions as described above (Scheme-2), however, it did not undergo cyclization to form the tricyclopentanoid systems 28/29 possibly again due to the resultant trans geometry at the junction after hydrogenation. If reduction of the double bond leads to a cis junction, it is possible to make use of the present methodology for the synthesis of tricyclopentanoids. In view of several tricyclopentanoids such as hirsutene, ${ }^{21}$ coriolic acid, ${ }^{22}$ isocomene $^{23}$ etc being important targets it is worth exploring the present synthetic strategy for their syntheses. Work towards this line is in progress. It is clear that the presence of an ester group in the original $\alpha$-hydroxy $-\beta, \gamma$-unsaturated esters is responsible for permitting the eventual Dieckman cyclization en-route to cyclopentanoids. We hope, therefore, that this strategy will be useful in organic synthesis.

Table 1. Isomerisation of Glycidic Esters with $\mathrm{Yb}(\mathrm{OTf})_{3}$ and with $\mathrm{Me}_{3} \mathrm{SiOTf}$

\begin{tabular}{|c|c|c|c|c|c|c|}
\hline \multirow[t]{2}{*}{ Entry } & \multirow[t]{2}{*}{ Glycidic ester } & \multirow{2}{*}{$\begin{array}{l}\alpha \text {-Hydroxy- } \beta, \gamma- \\
\text { unsaturated ester }\end{array}$} & \multicolumn{2}{|c|}{ Using $\mathrm{Yb}(\mathrm{OTf})_{3}$} & \multicolumn{2}{|c|}{ Using TMSOTf } \\
\hline & & & $\begin{array}{l}\text { Reaction } \\
\text { time }(\mathrm{h})\end{array}$ & $\begin{array}{l}\text { Yield } \\
(\%)\end{array}$ & $\begin{array}{l}\text { Reaction } \\
\text { time }(\mathrm{h})\end{array}$ & $\begin{array}{l}\text { Yield } \\
(\%)\end{array}$ \\
\hline 1 & $7^{6}$ & $7 a^{6}$ & 12 & 70 & 1 & 60 \\
\hline & $8^{6}$ & $8 a^{6}$ & 12 & 80 & 1 & 75 \\
\hline 4 & $10^{7}$ & $10 a^{7}$ & 24 & 82 & 1.5 & 80 \\
\hline
\end{tabular}




62

\section{Experimental Section}

General Procedures. Infrared spectra were recorded on Bruker FT/IR Vector 22 spectrometer as neat liquids. ${ }^{1} \mathrm{H}$ NMR were recorded on Jeol JNM-PMX 60, Bruker WP-80 and Jeol LA-400 NMR spectrometers using tetramethylsilane as an internal standard. ${ }^{13} \mathrm{C}$ NMR spectra were recorded on Jeol LA-400 NMR spectrometer in solution of $\mathrm{CDCl}_{3}$ using TMS as internal standard. Mass spectra were obtained using Jeol SX102/DA-6000 spectrometer. Elemental analysis were carried out using Thermo Quest EA 1110 (model) CE instruments C, H, N, O, S analyzer. Column chromatography was performed on silica gel (100-200 mesh, s.d.fine-chem Ltd. India). 
Glycidic esters. Compounds $7^{6}, \mathbf{8}^{6}, \mathbf{9}^{6}, \mathbf{1 0} \mathbf{1}^{7}, \mathbf{1 1}{ }^{7}, \mathbf{1 2}, \mathbf{1 3 ^ { 7 }}, \mathbf{1 4 ^ { 6 }}, \mathbf{1 5 ^ { 1 6 }}, \mathbf{1 6 ^ { 1 7 }}$ are known in the literature and were prepared according to the reported procedures. ${ }^{2}$,
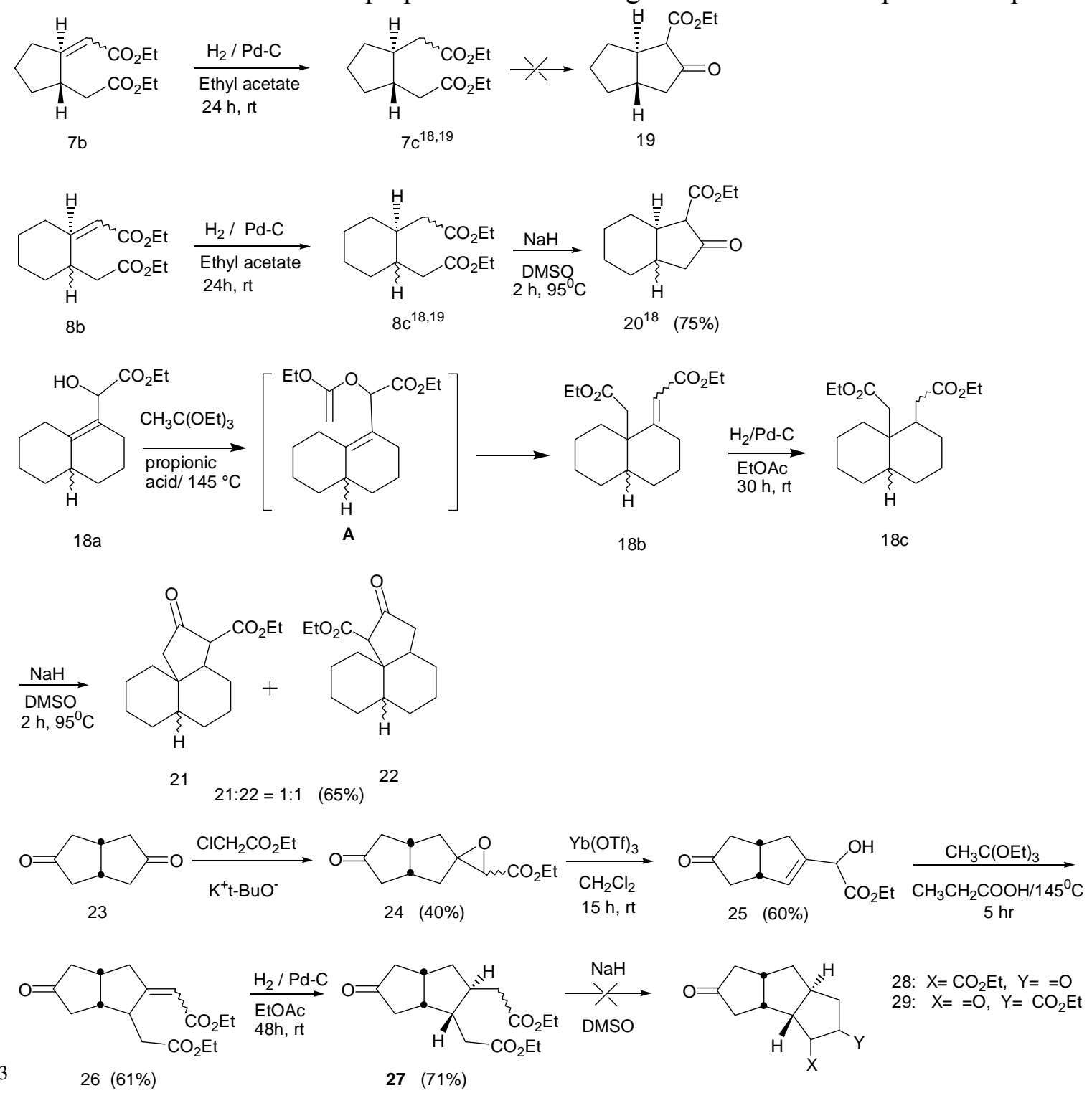

\section{Scheme 2}

3-Ethyl-3-phenyl-oxirane-2-carboxylic acid ethyl ester (17). Yield: 50\%. IR (neat) $v_{\max }$ : 1750, $1730 \mathrm{~cm}^{-1}$. ${ }^{1} \mathrm{H} \mathrm{NMR}\left(\mathrm{CCl}_{4}, 60 \mathrm{MHz}\right): \delta 0.8$ and $0.93(2 \mathrm{t}, 3 \mathrm{H}, \mathrm{J}=7,7 \mathrm{~Hz}), 1.3(\mathrm{t}, 3 \mathrm{H}, \mathrm{J}=8$ $\mathrm{Hz}) 1.6-2.43(\mathrm{~m}, 2 \mathrm{H}), 3.13$ and $3.53(2 \mathrm{~s}, 1 \mathrm{H}), 3.8$ and $4.26(2 \mathrm{q}, 2 \mathrm{H}, \mathrm{J}=7,6.5 \mathrm{~Hz}), 7.3(\mathrm{~s}, 5 \mathrm{H})$. MS (m/z): $220\left(\mathrm{M}^{+}\right)$. Anal. Calcd. for $\mathrm{C}_{13} \mathrm{H}_{16} \mathrm{O}_{3}$ : C, 70.89; H, 7.32\%. Found: C, 70.79; H, 7.14\%. Glycidic ester 24. IR (neat) $v_{\max }: 1752,1730, \mathrm{~cm}^{-1} .{ }^{1} \mathrm{H} \mathrm{NMR}\left(\mathrm{CDCl}_{3}, 400 \mathrm{MHz}\right): \delta 1.31$ (m, $3 \mathrm{H}), 1.64-1.82(\mathrm{~m}, 2 \mathrm{H}), 2.19-2.65(2 \mathrm{~m}, 6 \mathrm{H}), 2.75-2.9(\mathrm{~m}, 2 \mathrm{H}), 3.50(\mathrm{~s}, 1 \mathrm{H}), 4.11-4.30(\mathrm{~m}, 2 \mathrm{H})$. ${ }^{13} \mathrm{C} \mathrm{NMR}\left(\mathrm{CDCl}_{3}, 100 \mathrm{MHz}\right)$ : 14.16, 35.00, 38.80, 38.96, 43.56, 43.74, 55.66, 61.45, 70.20, 
115.97, 168.46, 219.03. Anal. Calcd. for $\mathrm{C}_{12} \mathrm{H}_{16} \mathrm{O}_{4}$ : C, 64.27; H, 7.19\%. Found: C, 64.23; H, $7.11 \%$.

Table 2. Johnson-Claisen Rearrangement of $\alpha$-hydroxy- $\beta$, $\gamma$-unsaturated Esters

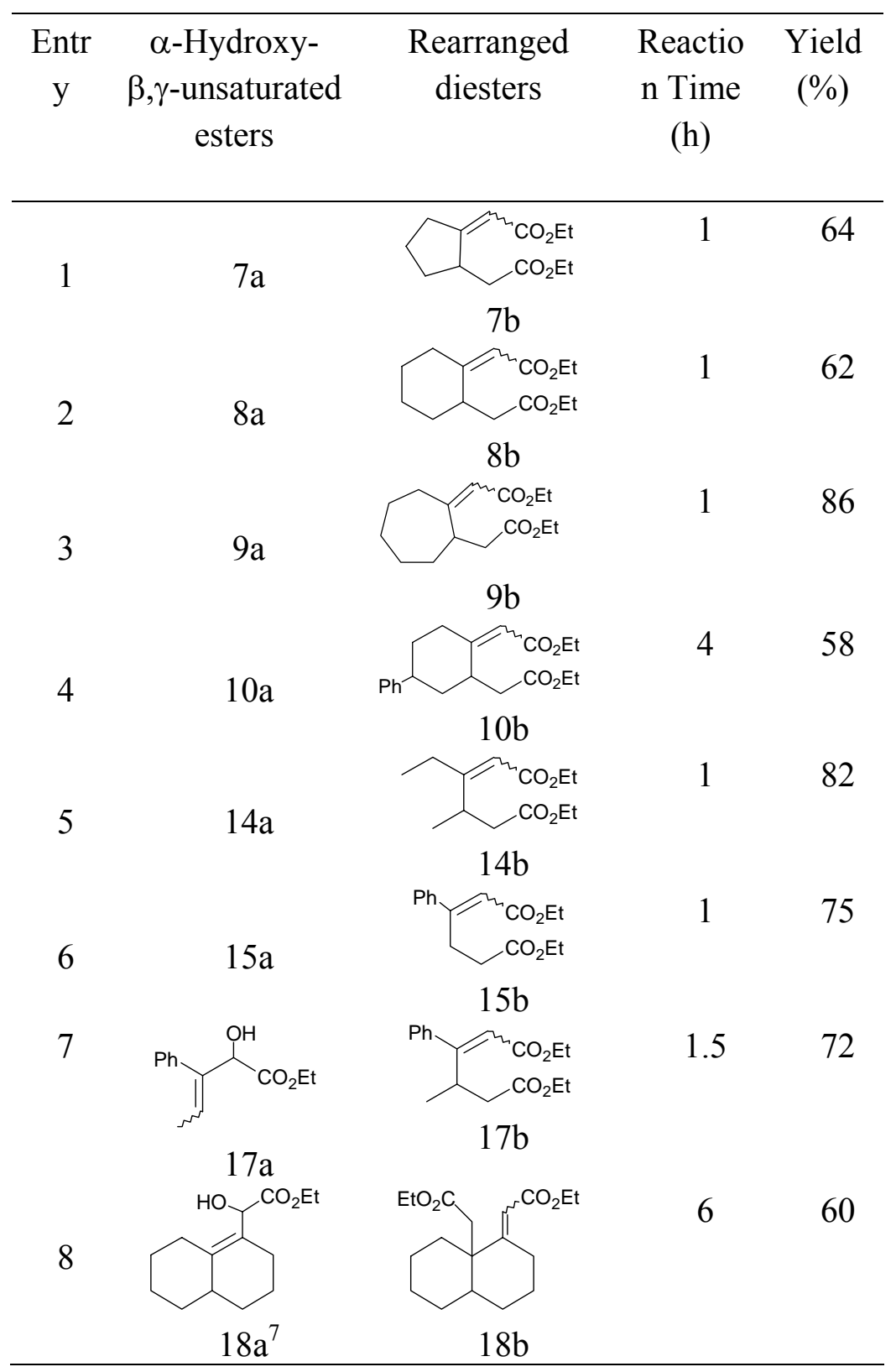

\section{Isomerisation of Glycidic esters to $\alpha$-Hydroxy- $\beta, \gamma$-unsaturated esters}

\section{General procedure}

using $\mathbf{Y b}(\mathbf{O T f})_{3}$ : To a stirred solution of a glycidic ester $(1 \mathrm{mmol})$ in dry $\mathrm{CH}_{2} \mathrm{Cl}_{2}(2 \mathrm{~mL})$ was added a catalytic amount of $\mathrm{Yb}(\mathrm{OTf})_{3}(15 \mathrm{mg})$ under $\mathrm{N}_{2}$ at room temperature and stirring continued. After the reaction was complete (TLC monitoring), the reaction mixture was 
quenched with saturated $\mathrm{NaHCO}_{3}(10 \mathrm{~mL})$ and extracted with $\mathrm{CH}_{2} \mathrm{Cl}_{2}(3 \times 15 \mathrm{~mL})$. The combined organic layer was washed with water $(2 \times 10 \mathrm{~mL})$, brine $(1 \times 10 \mathrm{~mL})$ and dried over anhydrous $\mathrm{Na}_{2} \mathrm{SO}_{4}$. Removal of solvent gave a crude product, which was purified by column chromatography $\left(\mathrm{SiO}_{2}\right)$.

Using TMSOTf: To a solution of a glycidic ester $(1 \mathrm{mmol})$ in dry $\mathrm{CH}_{2} \mathrm{Cl}_{2}(2 \mathrm{~mL})$ was added TMSOTf in catalytic amount ( 1 drop) under $\mathrm{N}_{2}$ at $-40^{\circ} \mathrm{C}$. The reaction mixture was stirred at that temperature and progress of the reaction was monitored by TLC. The reaction mixture was quenched by $\mathrm{NaHCO}_{3}$ solution $(10 \mathrm{~mL})$ and extracted with $\mathrm{CH}_{2} \mathrm{Cl}_{2}(3$ x $20 \mathrm{~mL})$. Usual work-up gave a crude product, which was purified by column chromatography $\left(\mathrm{SiO}_{2}\right)$.

Compounds $7 \mathbf{a}^{6}, \mathbf{8} \mathbf{a}^{6}, \mathbf{9} \mathbf{a}^{6}, \mathbf{1 0} \mathbf{a}^{7}, \mathbf{1 1} \mathbf{a}^{7}, \mathbf{1 2} \mathbf{a}^{8}, \mathbf{1 3} \mathbf{a}^{15}, \mathbf{1 4} \mathbf{a}^{6}, \mathbf{1 5} \mathbf{a}^{16}$ are known in the literature and their spectral and physical data were comparable with the compounds obtained by us.

2-Hydroxy-3-p-tolyl-but-3-enoic acid ethyl ester (16a). IR (neat) $v_{\max }: 3500,1720 \mathrm{~cm}^{-1}$. ${ }^{1} \mathrm{H}$ NMR $\left(\mathrm{CCl}_{4}, 60 \mathrm{MHz}\right): \delta 1.26(\mathrm{t}, 3 \mathrm{H}, \mathrm{J}=7 \mathrm{~Hz}), 2.46$ (s, 3H), 3.29 (brs, 1H), 4.06-4.39 (q, 2H, J $=7 \mathrm{~Hz}), 5.0(\mathrm{~s}, 1 \mathrm{H}), 5.46(\mathrm{~s}, 2 \mathrm{H}), 7.0-7.52(\mathrm{~m}, 4 \mathrm{H})$. Anal. Calcd. for $\mathrm{C}_{13} \mathrm{H}_{16} \mathrm{O}_{3}: \mathrm{C}, 70.89, \mathrm{H}$, 7.32\%. Found: C, 70.78; H, 7.26\%.

2-Hydroxy-3-phenyl-pent-3-enoic acid ethyl ester (17a). Yield: 65\%. IR (neat) $v_{\max }$ : 3500, $1720 \mathrm{~cm}^{-1}$. ${ }^{1} \mathrm{H} \mathrm{NMR}\left(\mathrm{CCl}_{4}, 60 \mathrm{MHz}\right): \delta 0.9$ and $1.2(2 \mathrm{t}, 3 \mathrm{H}, \mathrm{J}=7 \mathrm{~Hz}), 1.57$ and $1.87(2 \mathrm{~d}, 3 \mathrm{H}, \mathrm{J}$ $=7,7 \mathrm{~Hz}), 3.4$ (brs, 1H), $4.0(\mathrm{q}, 2 \mathrm{H}, \mathrm{J}=7 \mathrm{~Hz}), 4.63$ and $5.13(2 \mathrm{brs}, 1 \mathrm{H}), 5.83(\mathrm{q}, 1 \mathrm{H}, \mathrm{J}=7 \mathrm{~Hz})$, 7.13 (s, 5H). Anal. Calcd. for $\mathrm{C}_{13} \mathrm{H}_{16} \mathrm{O}_{3}$ : C, 70.89, H, 7.32\%. Found: C, 70.81; H, 7.19\%.

Hydroxy-(5-oxo-1,3a,4,5,6,6a-hexahydro-pentalen-2-yl)-acetic acid ethyl ester (25). IR (neat) $v_{\max }: 3470,1720, \mathrm{~cm}^{-1} .{ }^{1} \mathrm{H} \mathrm{NMR}\left(\mathrm{CDCl}_{3}, 400 \mathrm{MHz}\right): \delta 1.27-1.32(\mathrm{~m}, 3 \mathrm{H}), 1.90-2.12(\mathrm{~m}, 2 \mathrm{H})$, 2.2-2.55 (m, 4H), 2.75-3.10 (m, 2H), 3.48 (brs, 1H), 4.21-4.32 (m, 2H), 4.7 (brs, 1H,), 5.72 (s, $1 \mathrm{H}) .{ }^{13} \mathrm{C} \mathrm{NMR}\left(\mathrm{CDCl}_{3}, 100 \mathrm{MHz}\right): \delta 14.20,37.67,38.96,42.27,44.63,45.99,62.19,70.05$, 132.34, 140.75, 172.92, 219.44. Anal. Calcd. for $\mathrm{C}_{12} \mathrm{H}_{16} \mathrm{O}_{4}$ : C, 64.27; H, 7.19\%. Found: C, $64.20 ; \mathrm{H}, 7.08 \%$.

$\alpha, \beta$-Unsaturated esters after the Johnson-Claisen rearrangement. The experimental procedure was followed as reported in the literature. ${ }^{13}$

(2-Ethoxycarbonylmethylene-cyclopentyl)-acetic acid ethyl ester (7b). IR (neat) $v_{\max }$ : 1720, 1700, $1640 \mathrm{~cm}^{-1}$. ${ }^{1} \mathrm{H}$ NMR $\left(\mathrm{CCl}_{4}, 60 \mathrm{MHz}\right): \delta 1.26(\mathrm{t}, 6 \mathrm{H}, \mathrm{J}=7 \mathrm{~Hz}), 1.53-2.18(\mathrm{~m}, 4 \mathrm{H}), 2.43(\mathrm{~d}$, $2 \mathrm{H}, \mathrm{J}=6 \mathrm{~Hz}$ ), 2.6-3.17 (m, 3H), 4.1 (q, 4H, J = 7 Hz), 5.63 (brs, 1H). MS (m/z): $240\left(\mathrm{M}^{+}\right), 194$ $\left(\mathrm{M}^{+}\right.$- 46), $167\left(\mathrm{M}^{+}-73\right), 165\left(\mathrm{M}^{+}\right.$- 75). Anal. Calcd. for $\mathrm{C}_{13} \mathrm{H}_{20} \mathrm{O}_{4}$ : C, 64.98; $\mathrm{H}, 8.39 \%$. Found: C, 64.89\%; H, 8.34\%.

(2-Ethoxycarbonylmethylene-cyclohexyl)-acetic acid ethyl ester (8b). IR (neat) $v_{\max }$ : 1725, 1705, $1635 \mathrm{~cm}^{-1} .{ }^{1} \mathrm{H} \mathrm{NMR}\left(\mathrm{CCl}_{4}, 60 \mathrm{MHz}\right): \delta 1.23(\mathrm{t}, 6 \mathrm{H}, \mathrm{J}=7 \mathrm{~Hz}), 1.46-2.0(\mathrm{~m}, 6 \mathrm{H}), 2.2-2.93$ $(\mathrm{m}, 4 \mathrm{H}), 2.93-3.5(\mathrm{~m}, 1 \mathrm{H}), 4.1(\mathrm{q}, 4 \mathrm{H}, \mathrm{J}=7 \mathrm{~Hz}), 5.5(\mathrm{~s}, 1 \mathrm{H}) . \quad \mathrm{MS}(\mathrm{m} / \mathrm{z}): 254\left(\mathrm{M}^{+}\right), 208\left(\mathrm{M}^{+}\right.$46), $179\left(\mathrm{M}^{+}-75\right)$. Anal. Calcd. for $\mathrm{C}_{14} \mathrm{H}_{22} \mathrm{O}_{4}$ : C, 66.12\%; H, 8.72\%. Found: $\mathrm{C}, 66.04 \%$; $\mathrm{H}$, $8.73 \%$.

(2-Ethoxycarbonylmethylene-cycloheptyl)-acetic acid ethyl ester (9b). IR (neat) $v_{\max }$ : 1720, 1700, $1635 \mathrm{~cm}^{-1} .{ }^{1} \mathrm{H}$ NMR $\left(\mathrm{CCl}_{4}, 60 \mathrm{MHz}\right): \delta 1.25(\mathrm{t}, 6 \mathrm{H}, \mathrm{J}=7 \mathrm{~Hz}), 1.46-2.13(\mathrm{~m}, 8 \mathrm{H}), 2.3(\mathrm{~d}$, 
2H), 2.53-2.97 (m, 2H),2.97-3.45 (m, 1H), 4.1 (q, 4H, J = 7 Hz), 5.65 (brs, 1H). MS (m/z): 268 $\left(\mathrm{M}^{+}\right), 222\left(\mathrm{M}^{+}-46\right), 195\left(\mathrm{M}^{+}-73\right), 193\left(\mathrm{M}^{+}-75\right)$. Anal. Calcd. for $\mathrm{C}_{15} \mathrm{H}_{24} \mathrm{O}_{4}: \mathrm{C}, 67.14 ; \mathrm{H}, 9.01 \%$. Found: C, 67.09; H, 8.91\%.

(2-Ethoxycarbonylmethylene-5-phenyl-cyclohexyl)-acetic acid ethyl ester (10b). IR (neat) $v_{\max }: 1705,1720,1630 \mathrm{~cm}^{-1} .{ }^{1} \mathrm{H} \mathrm{NMR}\left(\mathrm{CCl}_{4}, 60 \mathrm{MHz}\right): \delta 1.22(\mathrm{t}, 6 \mathrm{H}, \mathrm{J}=7 \mathrm{~Hz}), 1.6-2.28(\mathrm{~m}$, $6 \mathrm{H}), 2.6(\mathrm{~d}, 2 \mathrm{H}), 2.73-3.26(\mathrm{~m}, 2 \mathrm{H}), 4.13(\mathrm{q}, 4 \mathrm{H}, \mathrm{J}=7 \mathrm{~Hz}), 5.3$ (brs, 1H), $7.16(\mathrm{~s}, 5 \mathrm{H})$. MS $(\mathrm{m} / \mathrm{z}): 330\left(\mathrm{M}^{+}\right), 284\left(\mathrm{M}^{+}-46\right), 256\left(\mathrm{M}^{+}-74\right), 255\left(\mathrm{M}^{+}-75\right)$. Anal. Calcd. for $\mathrm{C}_{20} \mathrm{H}_{26} \mathrm{O}_{4} \mathrm{C}, 72.70$; H, 7.93\%. Found: C, 72.66; H, 7.83\%.

3-Ethyl-4-methyl-hex-2-enedioic acid diethyl ester (14b). IR (neat) $v_{\max }: 1720,1635 \mathrm{~cm}^{-1} .{ }^{1} \mathrm{H}$ NMR $\left(\mathrm{CCl}_{4}, 60 \mathrm{MHz}\right): \delta$ 0.77-1.5 (m, 12H), 2.0-2.9 (m, 5H), 3.7-4.33 (m, 4H), $5.57(\mathrm{~s}, 1 \mathrm{H}) . \mathrm{MS}$ (m/z): $242\left(\mathrm{M}^{+}\right), 169\left(\mathrm{M}^{+}-73\right)$. Anal. Calcd. for $\mathrm{C}_{13} \mathrm{H}_{22} \mathrm{O}_{4}$ : C, 64.44; H, 9.15\%. Found: C, 64.35; H, $9.09 \%$.

3-Phenyl-hex-2-enedioic acid diethyl ester (15b). IR (neat) $v_{\max }: 1705,1720,1620 \mathrm{~cm}^{-1}$. ${ }^{1} \mathrm{H}$ NMR $\left(\mathrm{CCl}_{4}, 60 \mathrm{MHz}\right): \delta 1.2$ and $1.3(2 \mathrm{t}, 6 \mathrm{H}, \mathrm{J}=7,7 \mathrm{~Hz}), 2.36(\mathrm{t}, 2 \mathrm{H}, \mathrm{J}=8 \mathrm{~Hz}), 3.36(\mathrm{t}, 2 \mathrm{H}, \mathrm{J}=$ $8 \mathrm{~Hz}), 3.8-4.4(\mathrm{~m}, 4 \mathrm{H}), 6.0(\mathrm{~s}, 1 \mathrm{H}), 7.33(\mathrm{~s}, 5 \mathrm{H}) . \mathrm{MS}(\mathrm{m} / \mathrm{z}): 276\left(\mathrm{M}^{+}\right), 203\left(\mathrm{M}^{+}-73\right)$. Anal. Calcd. for $\mathrm{C}_{16} \mathrm{H}_{20} \mathrm{O}_{4}$ : C, 69.55; H, 7.29\%. Found: C, 69.49; H, 7.20\%.

4-Methyl-3-phenyl-hex-2-enedioic acid diethyl ester (17b). IR (neat) $v_{\max }$ : 1710, 1720, 1635, $1625 \mathrm{~cm}^{-1}$. ${ }^{1} \mathrm{H}$ NMR $\left(\mathrm{CCl}_{4}, 60 \mathrm{MHz}\right): \delta$ 0.8-1.5 (m, 9H), 2.23, $2.36(2 \mathrm{~d}, 2 \mathrm{H}, \mathrm{J}=6 \mathrm{~Hz}), 2.8-3.26$ $(\mathrm{m}, 1 \mathrm{H}), 3.6-4.43(\mathrm{~m}, 4 \mathrm{H}), 5.7$ and $5.76(2 \mathrm{~s}, 1 \mathrm{H}), 6.93-7.42(\mathrm{~m}, 5 \mathrm{H}) . \mathrm{MS}(\mathrm{m} / \mathrm{z}): 290\left(\mathrm{M}^{+}\right), 275$ $\left(\mathrm{M}^{+}-15\right), 217\left(\mathrm{M}^{+}-73\right)$. Anal. Calcd. for $\mathrm{C}_{17} \mathrm{H}_{22} \mathrm{O}_{4}$ : C, 70.32; H, 7.64\%. Found: C, 70.22; H, $7.58 \%$.

(8a-Ethoxycarbonylmethyl-octahydro-naphthalen-1-ylidene)-acetic acid ethyl ester (18b). IR (neat) $v_{\max }: 1700,1725,1620 \mathrm{~cm}^{-1} .{ }^{1} \mathrm{H} \mathrm{NMR}\left(\mathrm{CCl}_{4}, 60 \mathrm{MHz}\right): \delta 0.77-2.0(\mathrm{~m}, 19 \mathrm{H}), 2.13(\mathrm{~m}$, 2H), 2.23-3.0 (m, 2H, J = $8 \mathrm{~Hz}$ ), 4.1 (q, 4H, J = 7 Hz), 5.32 (brs, $1 \mathrm{H}) . \mathrm{MS}(\mathrm{m} / \mathrm{z}): 308\left(\mathrm{M}^{+}\right), 235$ $\left(\mathrm{M}^{+}\right.$- 73). Anal. Calcd. for $\mathrm{C}_{18} \mathrm{H}_{28} \mathrm{O}_{4}$ : C, 70.10; H, 9.15\%. Found: C, 69.99; H, 9.16\%.

(1-Ethoxycarbonylmethyl-5-oxo-hexahydro-pentalen-2-ylidene)-acetic acid ethyl ester (26). IR (neat) $v_{\max }: 1720,1700,1640 \mathrm{~cm}^{-1} .{ }^{1} \mathrm{H}$ NMR $\left(\mathrm{CDCl}_{3}, 400 \mathrm{MHz}\right): \delta 1.18-1.32$ (br t, $6 \mathrm{H}, \mathrm{J}=7$ $\mathrm{Hz}), 2.00-2.35$ (m, 4H), 2.36-2.65 (m, 5H), 2.75-3.00 (m, 2H), 3.15-3.37 (m, 1H), 4.04-4.23 (br $\mathrm{q}, 4 \mathrm{H}, \mathrm{J}=7 \mathrm{~Hz}) .{ }^{13} \mathrm{C} \mathrm{NMR}\left(\mathrm{CDCl}_{3}, 100 \mathrm{MHz}\right)$ : (peaks for the major diastereomer) $\delta 218.61$, 171.4, 167.6, 166.30, 114.6, 60.78, 59.87, 47.80, 45.14, 43.85, 42.87, 38.49, 38.27, 37.44, 14.26, 14.17. $\mathrm{MS}(\mathrm{m} / \mathrm{z}): 295\left(\mathrm{M}^{+}\right), 207\left(\mathrm{M}^{+}-88\right)$. Anal. Calcd. for $\mathrm{C}_{16} \mathrm{H}_{22} \mathrm{O}_{5}: \mathrm{C}, 65.29 ; \mathrm{H}, 7.53 \%$. Found; C, 65.21; H, 7.45\%.

\section{Saturated diesters after hydrogenation}

(2-Ethoxycarbonylmethyl-cyclopentyl)-acetic acid ethyl ester (7c). IR (neat) $v_{\max }: 1725 \mathrm{~cm}^{-1}$. ${ }^{1} \mathrm{H}$ NMR $\left(\mathrm{CDCl}_{3}, 400 \mathrm{MHz}\right): \delta 1.25(\mathrm{t}, 6 \mathrm{H}, \mathrm{J}=7.1 \mathrm{~Hz}), 1.18-1.95(\mathrm{~m}, 8 \mathrm{H}), 2.19(\mathrm{dd}, 2 \mathrm{H}, \mathrm{J}=8.0$ $\mathrm{Hz}, 15.1 \mathrm{~Hz}), 2.47$ (dd, 2H, $3.9 \mathrm{~Hz}, 15.1 \mathrm{~Hz}), 4.13$ (q, 4H, J =7.1 Hz). ${ }^{13} \mathrm{C} \mathrm{NMR}\left(\mathrm{CDCl}_{3}, 100\right.$ $\mathrm{MHz}$ ): $\delta$ (trans isomer): 172.88, 60.01, 39.09, 34.99, 31.93, 23.16, 14.05. (cis isomer):172.99, 60.08, 38.61, 34.35, 30.20, 21.99, 14.05. Anal. Calcd. for $\mathrm{C}_{13} \mathrm{H}_{22} \mathrm{O}_{4}$ : C, 64.43; H, 9.15\%. Found: C, $64.33 ; \mathrm{H}, 9.06 \%$. 
(2-Ethoxycarbonylmethyl-cyclohexyl)-acetic acid ethyl ester (8c). IR (neat) $v_{\max }: 1725 \mathrm{~cm}^{-1}$. ${ }^{1} \mathrm{H}$ NMR $\left(\mathrm{CDCl}_{3}, 400 \mathrm{MHz}\right): \delta 1.08-1.8(\mathrm{~m}, 10 \mathrm{H}), 1.26(\mathrm{t}, 6 \mathrm{H}, \mathrm{J}=7.0 \mathrm{~Hz}), 2.1(\mathrm{dd}, 1 \mathrm{H}, \mathrm{J}=7.8$ $\mathrm{Hz}, 14.9 \mathrm{~Hz}), 2.15-2.3(\mathrm{~m}, 2 \mathrm{H}), 2.48(\mathrm{dd}, 1 \mathrm{H}, \mathrm{J}=3.4 \mathrm{~Hz}, 14.9 \mathrm{~Hz}), 4.13(\mathrm{q}, 4 \mathrm{H}, \mathrm{J}=7.0 \mathrm{~Hz}) .{ }^{13} \mathrm{C}$ NMR ( $\left.\mathrm{CDCl}_{3}, 100 \mathrm{MHz}\right): \delta$ (trans isomer): 173.17, 60.23, 39.21, 35.67, 32.29, 25.74, 14.21. (cis isomer): 173.2, 60.27, 39.02, 35.47, 28.72, 23.04, 14.21. MS (m/z): $210\left(\mathrm{M}^{+}-46\right)$. Anal. Calcd. for $\mathrm{C}_{14} \mathrm{H}_{24} \mathrm{O}_{4}$ : C,65.60; $\mathrm{H}, 9.44 \%$. Found: $\mathrm{C}, 65.63 ; \mathrm{H}, 9.36 \%$.

(8a-Ethoxycarbonylmethyl-decahydro-naphthalen-1-yl)-acetic acid ethyl ester (18c). IR (neat) $v_{\max }: 1725 \mathrm{~cm}^{-1} .{ }^{1} \mathrm{H}$ NMR $\left(\mathrm{CDCl}_{3}, 400 \mathrm{MHz}\right): \delta 1.8-2.0(\mathrm{~m}, 16 \mathrm{H}), 1.25(\mathrm{t}, 6 \mathrm{H}, \mathrm{J}=7 \mathrm{~Hz})$, 2.06-2.47 (m, 4H), 4.08 (q, 4H, J = 7 Hz). MS (m/z): $310\left(\mathrm{M}^{+}\right), 237\left(\mathrm{M}^{+}-73\right)$. Anal. Calcd. for $\mathrm{C}_{18} \mathrm{H}_{30} \mathrm{O}_{4}$ : C, 69.64; H, 9.74\%. Found: C, 69.59; H, 9.63\%.

(1-Ethoxycarbonylmethyl-5-oxo-octahydro-pentalen-2-yl)-acetic acid ethyl ester (27). IR (neat) $v_{\max }: 1740 \mathrm{~cm}^{-1} .{ }^{1} \mathrm{H}$ NMR $\left(\mathrm{CDCl}_{3}, 400 \mathrm{MHz}\right): \delta 1.05-1.27$ (br t, $\left.6 \mathrm{H}, \mathrm{J}=7 \mathrm{~Hz}\right), 2.0-2.18$ (m, 4H), 2.2-2.38 (m, 4H), 2.44-2.6 (m, 4H), 2.62-2.96 (m, 1H), 3.00-3.20 (m, 1H), 4.2-4.2 (m, $4 \mathrm{H}) .{ }^{13} \mathrm{C} \mathrm{NMR}\left(\mathrm{CDCl}_{3}, 100 \mathrm{MHz}\right)$ : (peaks for the major diastereomer) $\delta 219.63,172.42,172.31$, $60.35,60.25,45.37,44.31,43.88,43.14,39.83,37.67,37.50,37.34,32.70,14.14,14.08$. MS (m/z): 269 [( $\left.\left.\mathrm{M}^{+}+1\right)-28\right], 182\left(\mathrm{M}^{+}-116\right)$. Anal. Calcd. for $\mathrm{C}_{16} \mathrm{H}_{24} \mathrm{O}_{5}: \mathrm{C}, 64.85 ; \mathrm{H}, 8.16 \%$, Found: C, 64.80; H, 8.11\%.

\section{General procedure for Dieckman cyclization ${ }^{14}$}

A solution of a saturated diester $(2 \mathrm{mmol})$ in DMSO $(2 \mathrm{~mL})$ was added to $\mathrm{NaH}(4 \mathrm{mmol})$ in DMSO $(2 \mathrm{~mL})$ and the resulting mixture was stirred at $95^{\circ} \mathrm{C}$ under $\mathrm{N}_{2}$ atmosphere. After completion of the reaction (TLC monitoring), it was cooled to room temperature and quenched with $2 \mathrm{~mL}$ of saturated aqueous $\mathrm{NH}_{4} \mathrm{Cl}$ solution. Usual work-up gave the crude product which was purified by column chromatography.

2-Oxo-octahydro-indene-1-carboxylic acid ethyl ester (20). IR (neat) $v_{\max }: 1735,1710 \mathrm{~cm}^{-1}$. ${ }^{1} \mathrm{H} \mathrm{NMR}\left(\mathrm{CDCl}_{3}, 400 \mathrm{MHz}\right): \delta 1.23(\mathrm{t}, 3 \mathrm{H}, \mathrm{J}=7.0 \mathrm{~Hz}), 1.1-2.0(\mathrm{~m}, 8 \mathrm{H}), 2.35-2.45(\mathrm{~m}, 2 \mathrm{H})$, $2.80(\mathrm{~d}, 2 \mathrm{H}, \mathrm{J}=12.2 \mathrm{~Hz}), 3.13(\mathrm{~d}, 1 \mathrm{H}, \mathrm{J}=10.8 \mathrm{~Hz}), 4.15(\mathrm{q}, 2 \mathrm{H}, \mathrm{J}=7.0 \mathrm{~Hz}) . \mathrm{MS}(\mathrm{m} / \mathrm{z}): 210$ $\left(\mathrm{M}^{+}\right), 169\left(\mathrm{M}^{+}-41\right), 139\left(\mathrm{M}^{+}-69\right)$. Anal. Calcd. for $\mathrm{C}_{12} \mathrm{H}_{18} \mathrm{O}_{3}$ : C, 68.55; $\mathrm{H}, 8.63 \%$. Found: $\mathrm{C}$, $68.48 ; \mathrm{H}, 8.59 \%$.

2-Oxo-dodecahydro-cyclopenta[d]naphthalene-3-carboxylic acid ethyl ester (21) and 2Oxo-dodecahydro-cyclopenta[d]naphthalene-1-carboxylic acid ethyl ester (22). IR (neat) $v_{\text {max }}: 1730,1710 \mathrm{~cm}^{-1}$. ${ }^{1} \mathrm{H}$ NMR $\left(\mathrm{CDCl}_{3}, 400 \mathrm{MHz}\right): \delta 1.2-2.1(\mathrm{~m}, 18 \mathrm{H}), 2.1-2.6$ and 2.77-3.03 (2m, 3H), 2.77 and $3.03(2 \mathrm{~d}, 2 \mathrm{H}, \mathrm{J}=13 \mathrm{~Hz}), 3.26-3.48(2 \mathrm{~m}, 1 \mathrm{H}), 4.15-4.26(\mathrm{~m}, 2 \mathrm{H}) . \mathrm{MS}(\mathrm{m} / \mathrm{z})$ : $264\left(\mathrm{M}^{+}\right)$. Anal. Calcd. for $\mathrm{C}_{14} \mathrm{H}_{24} \mathrm{O}_{3}$ : C, 72.69; H, 9.15\%. Found: C, 72.65; H, 9.18\%.

\section{Acknowledgements}

We thank the 'Council of Scientific and Industrial Research, New Delhi' for the financial assistance of a 'Research Associateship' to AG and a 'Junior Research Fellowship' to SR. We thank Dr. Ganesh Pandey for helpful discussions. 


\section{References and Notes}

1. Part 17, General Synthetic Methods, For part 16 see Rani, S.; Lokesh Babu, J.; Vankar, Y.D. J. Org. Chem (Submitted).

2. Lunt J. C.; Sondheimer K. J. Chem. Soc. 1950, 2957.

3. Johnson. W. S.; Belew J. S.; Chinn L. J.; Hunt R. H. J. Am. Chem. Soc. 1953, 75, 4995.

4. Camps, F.; Castells, J.; Pascual J. J. Org. Chem. 1966, 31, 3510.

5. Vankar, Y.D.; Chaudhuri, N.C.; Vankar P.S. J. Chem. Res. 1989, 178.

6. Hartmann B.C.; Rickborn, B. J. Org. Chem. 1972, 37, 943.

7. Reddy, M.V.R.; Pitre, S.V.; Bhattacharya, I.; Vankar, Y.D. Synlett 1996, 3, 241.

8. Hachoumy, M.; Mathew, T.; Tongco, E.C.; Vankar, Y.D.; Prakash, G.K.S.; Olah, G.A. Synlett 1999, 3, 363.

9. (a) Kobayashi, S. Synlett 1994, 413. (b) Yu, L.; Chen, D.; Wang, P.G. Tetrahedron Lett. 1996, 37, 2169.

10. Michelet, V.; Adiey, K.; Bulic, B.; Genet, J.P.; Dujardin, G.; Rossignol, S.; Brown, E.; Toupet L. Eur. J. Org. Chem. 1999, 2885.

11. Bhattacharya, I.; Vankar, P.S.; Shah, K.; Vankar, Y.D. Synth. Commun. 1993, 23, 2405.

12. Vankar, P.S.; Bhattacharya, I.; Vankar, Y.D. Tetrahedron: Asymmetry 1996, 7, 1683.

13. Johnson, W.S.; Werthmann, L.; Bartlett, W.R.; Brocksorn, J.J.; Lee, T.; D. Faulkner, D.J.; Peterson, M.R. J. Am. Chem. Soc. 1970, 92, 741.

14. Dieckmann, W. Ber 1894, 27(102), 965.

15. Vogel, E.; Schinz H. Helv. Chim. Acta, 1950, 33, 116.

16. Johnson, W.S.; Belew, J.S.; Madden D.A. J. Am. Chem. Soc. 1958, 80, 6386.

17. Hondak, T.; Kimura, N.; Tsubuki M. Tetrahedron: Asymmetry 1993, 4 (1), 21.

18. The $\%$ estimation of cis-trans isomers was done by capillary GC (30 m, polydimethyl siloxane $100 \mu \mathrm{m}$ film) and ${ }^{13} \mathrm{C} \mathrm{NMR}$ combination. In case of compound 7c, the ratio of cis/trans is 10/90 whereas in case of compound 8c it is 40/60. Pandey, G.; Ghorai, M.K.; Hajra, S. Tetrahedron Lett. 1998, 39, 1831. (b) De Riggi, I.; Surzur, J.-M.; Bertrand, M.P. Tetrahedron 1988, 44, 7119.

20. Bertz, S.H.; Cook, J.M.; Gawish, A.; Weiss, U. Org. Syn. Coll., vii, 1990, 50.

21. (a) Hua, D.H.; Venaktaraman, S.; Ostrander, R.A.; Sinai, G.-Z.; McCann, P.J.; Coulter, M.J.; Xu, M.R. J. Org. Chem. 1988, 53, 507. (b) Curran, D.P.; Raciewicz, D.M. J. Am. Chem. Soc. 1985, 107, 1448.

22. Shibasaki, M.; Iseki, K.; Ikegami, S. Tetrahedron Lett. 1980, 3587.

23. Snider, B.B.; Beal, R.B. J. Org. Chem. 1988, 53, 4508. 